\title{
Brain Connectivity Using Geodesics in HARDI
}

\author{
Mickaël Péchaud ${ }^{1,2}$, Maxime Descoteaux ${ }^{3}$, and Renaud Keriven ${ }^{2}$ \\ 1 LIENS, École Normale Supérieure \\ 2 IMAGINE, Université Paris Est \\ 3 Neurospin, IFR 49 CEA Saclay, France
}

\begin{abstract}
We develop an algorithm for brain connectivity assessment using geodesics in HARDI (high angular resolution diffusion imaging). We propose to recast the problem of finding fibers bundles and connectivity maps to the calculation of shortest paths on a Riemannian manifold defined from fiber ODFs computed from HARDI measurements. Several experiments on real data show that our method is able to segment fibers bundles that are not easily recovered by other existing methods.
\end{abstract}

\section{Introduction}

Diffusion MRI and fiber tractography have gained importance in the medical imaging community for the last decade. Many new diffusion models and fiber tracking algorithms have recently appeared in the literature always seeking better brain connectivity assessment, in particular regarding complex fiber configuration such as crossing, branching or kissing fibers. Clinical applications are also asking for robust tractography methods, as they are the unique in vivo tool to study the integrity of brain connectivity.

The most commonly used model is the diffusion tensor (DT), which is only able to characterize one fiber compartment per voxel. Several alternatives have been proposed to overcome this limitation of DTI, mainly using high angular resolution diffusion imaging (HARDI). Several competing HARDI reconstruction technique exist in the literature, which all have their advantages and disadvantages. Nonetheless, the community seems to now agree that a sharp orientation distribution function (ODF), often called fiber ODF or fiber orientation density function (fODF) 112 3/4, able to discriminate low angle crossing fibers needs to be used for fiber tractography.

Three classes of algorithms exist: deterministic, probabilistic and geodesic. A large number of tractography algorithms have been developed for DTI, which are limited in regions of fiber crossings. While HARDI-based extensions of streamline deterministic [5]6/7/4 and probabilistic 8 8/10/11/213/4 tracking algorithms have flourished in the last few years (the list is not exhaustive), 14] was the only attempt to generalize DTI geodesic tracking [15/16] for HARDI measurements.

In this paper, we develop an algorithm for brain connectivity assessment using geodesics in HARDI. We propose to recast the problem of finding connectivity maps in the white matter to the calculation of shortest paths on a Riemannian manifold. This Riemannian manifold is defined from fiber ODFs computed from HARDI measurements. 


\section{Method}

Firstly, let us provide some basics definitions about Riemannian manifolds.

Definitions. Let $(M, g)$ be a Riemannian manifold i.e.

- $M$ is a $n$-dimensional manifold

- for all $x \in M, g(x)$ is a symmetric positive definite $n \times n$ matrix inducing a metric $\|y\|_{x} \stackrel{\text { def. }}{=} \sqrt{y^{T} g^{-1}(x) y}$ over that manifold.

The length of a smooth curve $\gamma:[0,1] \rightarrow M$ is then defined as

$$
\mathcal{L}(\gamma) \stackrel{\text { def. }}{=} \int_{0}^{1}\left\|\gamma^{\prime}(t)\right\|_{\gamma(t)} \mathrm{d} t \stackrel{\text { def. }}{=} \int_{0}^{1} \sqrt{\gamma^{\prime}(t)^{T} g^{-1}(\gamma(t)) \gamma^{\prime}(t)} d t .
$$

Given a set $\mathcal{A} \subset M$ of seeds points and a set $\mathcal{B} \subset M$ of ending points, a geodesic $\gamma^{*}(t) \subset M$ joining $\mathcal{A}$ to $\mathcal{B}$ is defined as a curve with minimal length between $\mathcal{A}$ and $\mathcal{B}$ :

$$
\gamma^{*}(\mathcal{A}, \mathcal{B}) \stackrel{\text { def. }}{=} \underset{\gamma \in \pi(\mathcal{A}, \mathcal{B})}{\operatorname{argmin}} \mathcal{L}(\gamma)
$$

where $\pi(\mathcal{A}, \mathcal{B})$ is the set of curves $\gamma$ such that $\gamma(0) \in \mathcal{A}$ and $\gamma(1) \in \mathcal{B}$. The corresponding geodesic distance is $d(\mathcal{A}, \mathcal{B}) \stackrel{\text { def. }}{=} \mathcal{L}\left(\gamma^{*}(\mathcal{A}, \mathcal{B})\right)$.

Let us also define the Euclidean length of the curve $\gamma$

$$
\mathcal{L}_{\text {euc }}(\gamma) \stackrel{\text { def. }}{=} \int_{0}^{1}\left\|\gamma^{\prime}(t)\right\| \mathrm{d} t
$$

and

$$
\mathcal{L}_{s q}(\gamma) \stackrel{\text { def. }}{=} \int_{0}^{1}\left\|\gamma^{\prime}(t)\right\|_{\gamma(t)}^{2} \mathrm{~d} t
$$

Following [15] if we interpret the metric induced by $g$ as a "speed" over $M$, for any smooth curve $\gamma, \mathcal{L}(\gamma) / \mathcal{L}_{\text {euc }}(\gamma)$ can be thought of as the average of inverse speed along the curve, while $\sqrt{\mathcal{L}_{s q}(\gamma) / \mathcal{L}_{\text {euc }}(\gamma)-\left(\mathcal{L}(\gamma) / \mathcal{L}_{\text {euc }}(\gamma)\right)^{2}}$ represents the standard deviation of this quantity.

Connectivity measures. Considering $\mathcal{A}$ and $\mathcal{B}$ two subset of $M$ we define

$$
\begin{aligned}
& \mathcal{C}(\mathcal{A}, \mathcal{B}) \stackrel{\text { def. }}{=} \frac{\mathcal{L}\left(\gamma^{*}(\mathcal{A}, \mathcal{B})\right)}{\mathcal{L}_{\text {euc }}\left(\gamma^{*}(\mathcal{A}, \mathcal{B})\right)}, \quad \mathcal{C}_{\max }(\mathcal{A}, \mathcal{B}) \stackrel{\text { def. }}{=} \max _{t \in[0 . .1]}\left\|\left(\gamma^{*}(\mathcal{A}, \mathcal{B})\right)^{\prime}(t)\right\|_{\gamma(t)} \\
& \mathcal{C}_{\sigma}(\mathcal{A}, \mathcal{B}) \stackrel{\text { def. }}{=} \sqrt{\left(\frac{\mathcal{L}\left(\gamma^{*}(\mathcal{A}, \mathcal{B})\right)}{\mathcal{L}_{\text {euc }}\left(\gamma^{*}(\mathcal{A}, \mathcal{B})\right)}\right)^{2}-\frac{\mathcal{L}_{s q}\left(\gamma^{*}(\mathcal{A}, \mathcal{B})\right)}{\mathcal{L}_{\text {euc }}\left(\gamma^{*}(\mathcal{A}, \mathcal{B})\right)}}
\end{aligned}
$$

$\gamma^{*}(\mathcal{A}, \mathcal{B})$ being a geodesic between $\mathcal{A}$ and $\mathcal{B}, \mathcal{C}(\mathcal{A}, \mathcal{B}), \mathcal{C}_{\sigma}(\mathcal{A}, \mathcal{B})$ and $\mathcal{C}_{\text {max }}(\mathcal{A}, \mathcal{B})$ are respectively measures of average inverse speed, inverse speed standard deviation, and worst inverse speed to reach $\mathcal{B}$ from $\mathcal{A}$. They can thus be interpreted as three different connectivity measures between $\mathcal{A}$ and $\mathcal{B}$. 


\subsection{HARDI Riemannian Manifold}

We now explain how we recast the fibers bundles tracking problem from HARDI data to the calculation of connectivity maps on a Riemannian manifold.

Let us denote $E \subset \mathbb{R}^{3}$ the white matter volume, $\mathcal{S}=\left\{e_{\theta, \varphi} \mid \theta \in[0,2 \pi), \varphi \in\right.$ $[0, \pi)\}$ the unit sphere and $M \stackrel{\text { def. }}{=} E \times \mathcal{S}$. Using such a 5-dimensional space can disambiguate crossing configurations since in such a space $\left(x, y, z, e_{\theta, \varphi}\right)$ and $\left(x, y, z, e_{\theta^{\prime}, \varphi^{\prime}}\right)$ are completely different points. The idea was introduced [17, but the authors proposed to segment rather than track bundles using level-sets, which is time-consuming and less accurate.

At every point $(x, y, z) \in E$, we can compute the fODF $f_{x y z}: e_{\theta, \varphi} \in \mathcal{S} \rightarrow$ $f_{x y z}\left(e_{\theta, \varphi}\right) \in \mathbb{R}^{+}$. The full data can thus be naturally modelled as a mapping $f$ from $M$ to $\mathbb{R}^{+}: f:\left(x, y, z, e_{\theta, \varphi}\right) \in M \mapsto f_{x y z \theta \varphi} \stackrel{\text { def. }}{=} f_{x y z}\left(e_{\theta, \varphi}\right) \in \mathbb{R}^{+}$.

Let us define the metric $g$ at any point $\left(x, y, z, e_{\theta, \varphi}\right)$ of $M$ as

$$
g_{x y z \theta \varphi} \stackrel{\text { def. }}{=}\left(\begin{array}{cccccc}
\rho\left(f_{x y z \theta \varphi}\right) & 0 & 0 & 0 & 0 \\
0 & \rho\left(f_{x y z \theta \varphi}\right) & 0 & 0 & 0 \\
0 & 0 & 0 & 0 \\
\left.\hdashline f_{x y z} z \underline{\varphi}\right) & 0 & 0 \\
\hdashline 0 & 0 & 0 & \alpha & 0 \\
0 & 0 & 0 & 0 & \alpha
\end{array}\right)=\left(\begin{array}{ccc}
\rho\left(f_{x y z \theta \varphi}\right) I_{3} & 0 \\
0 & \alpha I_{2}
\end{array}\right)
$$

where $\rho$ is an increasing function from $\mathbb{R}^{+}$to $\mathbb{R}^{+*}$ and $\alpha$ is a parameter controlling the speed on the angular space $\mathcal{S}$ w.r.t. the speed on the $E$ volume. Such a metric "favors" paths going through areas of high diffusion.

Recasting the problem in the white matter volume, let us consider two points $\left(x_{1}, y_{1}, z_{1}\right)$ and $\left(x_{2}, y_{2}, z_{2}\right) \in E$ between which one wishes to estimate the connectivity. Let us denote $\mathcal{A}=\left\{x_{1}, y_{1}, z_{1}, e_{\theta, \varphi} \mid e_{\theta, \varphi} \in \mathcal{S}\right\}$ and $\mathcal{B}=$ $\left\{x_{2}, y_{2}, z_{2}, e_{\theta, \varphi} \mid e_{\theta, \varphi} \in \mathcal{S}\right\} \subset E \times \mathcal{S}$.

$\mathcal{C}(\mathcal{A}, \mathcal{B}), \mathcal{C}_{\sigma}(\mathcal{A}, \mathcal{B})$ and $\mathcal{C}_{\max }(\mathcal{A}, \mathcal{B})$ are then natural measures of connectivity between $\left(x_{1}, y_{1}, z_{1}\right)$ and $\left(x_{2}, y_{2}, z_{2}\right)$. Furthermore, let us denote $\pi: E \times \mathcal{S} \rightarrow E$ the projection such that $\pi\left(x, y, z, e_{\theta, \varphi}\right)=(x, y, z)$. To the geodesic $\gamma^{*}(\mathcal{A}, \mathcal{B})$ in $E \times \mathcal{S}$ then corresponds a projected path $\pi\left(\gamma^{*}(\mathcal{A}, \mathcal{B})\right)$ in $E \subset \mathbb{R}^{3}$. Since $\gamma^{*}(\mathcal{A}, \mathcal{B})$ follows a high diffusion trajectory, $\pi\left(\gamma^{*}(\mathcal{A}, \mathcal{B})\right)$ is likely to follow an actual fiber bundle in the volume. With this point of view, $\alpha$ can be seen as a smoothing parameter of the angular variations of the fibers.

However, among the paths $\gamma:[0,1] \rightarrow M$, one would like to favor the ones such that at every point $t_{0}, \pi\left(\gamma\left(t_{0}\right)\right)$ follows the corresponding direction in $\mathcal{S}$ : if we denote $\left(x_{0}, y_{0}, z_{0}, e_{\theta_{0}, \varphi_{0}}\right) \stackrel{\text { def. }}{=} \gamma\left(t_{0}\right)$, one would like to have

$$
\left(\pi(\gamma)_{x}\left(t_{0}\right), \pi(\gamma)_{y}\left(t_{0}\right), \pi(\gamma)_{z}\left(t_{0}\right)\right) \approx \pm e_{\theta_{0}, \varphi_{0}}\left\|\left(\pi(\gamma)_{x}\left(t_{0}\right), \pi(\gamma)_{y}\left(t_{0}\right), \pi(\gamma)_{z}\left(t_{0}\right)\right)\right\|
$$

In order to encourage these paths, we propose the following approach : let us consider a point $\left(x, y, z, e_{\theta, \varphi}\right)$. Instead of using an isotropic metric $\rho\left(f_{x y z \theta \varphi}\right) I_{3}$ in the first three directions, one would like to favor propagation along the $e_{\theta, \varphi}$ direction. In order to do so, $\rho\left(f_{x y z \theta \varphi}\right) I_{3}$ is replaced by the following matrix: 


$$
\left(R_{\theta, \varphi}\right)^{T}\left(\begin{array}{ccc}
\rho\left(f_{x y z \theta \varphi}\right) & 0 & 0 \\
0 & \min \left(\varepsilon, \rho\left(f_{x y z \theta \varphi}\right)\right) & 0 \\
0 & 0 & \min \left(\varepsilon, \rho\left(f_{x y z \theta \varphi}\right)\right)
\end{array}\right) R_{\theta, \varphi}
$$

where $R_{\theta, \varphi}$ is a rotation which maps the first axis to the $e_{\theta, \varphi}$ direction, and $\varepsilon$ is some constant. As long as $\rho\left(f_{x y z \theta \varphi}\right)>\varepsilon$, this tensor favors propagation in the $e_{\theta, \varphi}$ direction. However if $\rho\left(f_{x y z \theta \varphi}\right) \leqslant \varepsilon$ (i.e. if the diffusion is small at this point), this does not make sense, and we keep the isotropic tensor defined by $\rho\left(f_{x y z \theta \varphi}\right) I_{3}$.

The choice of this metric is a natural way of handling the 5-dimensional HARDI data and to obtain connectivity maps and fibers. It ensures that (i) the full HARDI angular information is used, (ii) geodesics go through areas of high diffusion, (iii) geodesics travel in those areas in the correct directions and (iv) crossing configurations are disambiguated.

\section{Implementation}

\subsection{Djikstra and Fast-Marching Algorithms}

Two algorithms can be used to compute connectivity measures on discretized Riemannian manifolds $(M, g)$. Assuming an initial seed $\mathcal{A} \subset M$, they both consist in successive evaluations of geodesic distances $d(\mathcal{A},\{x\})$ and connectivity measures from each point $x \in M$ to $\mathcal{A}$. For one point $x, d(\mathcal{A},\{x\})$ is iteratively evaluated from the $\{d(\mathcal{A},\{y\})\}_{y \in N(x)}$, where $N(x)$ is the set of neighbors of $x$ in the chosen discretization. This calculation is called local update step. Only this local update step differs between the two following methods.

- Djikstra algorithm-initially designed to compute distances and shortest paths in graphs-can be used to approximate connectivity maps and geodesics on Riemannian manifolds. While this algorithm is fast, paths are constrained to be on the edges on the discretization, which limits its accuracy.

- Fast-Marching algorithm 1819 and its variants can be view as a refinement of Djikstra algorithm in which the paths are not constrained anymore. However, while being of same asymptotic complexity, it is much slower than Djikstra algorithm, and thus can not be directly applied to our problem.

In most tracking methods, connectivity measures are obtained explicitly from fibers computed from deterministic or probabilistic streamlines. However, in Djikstra and Fast-Marching algorithms, the connectivity measures are computed intrinsically without the actual computation of any fiber, although the geodesics - i.e. the fibers - can be retrieved from the output of the algorithm by performing a gradient descent on the distance map.

\subsection{Our Implementation}

For our problem, $E$ was discretized as a subset of a 3 -dimensional grid, at the HARDI measurement spatial definition. $\mathcal{S}$ was meshed in such a way that every 
vertex of the mesh corresponds to a direction of HARDI measurements. Furthermore, in order to achieve good precision, we chose to use a 26-neighborhood in the discretization of $E$. Since we are mainly interested in precision in the high diffusion directions, we propose to compute $d(\mathcal{A},\{x\})$ at each point by using Djikstra local update step. The Fast-Marching local update step is then only applied for neighbors near to the current $e_{\theta, \varphi}$ direction, and only if the diffusion is important enough (i.e. $\rho\left(f_{x y z \theta \varphi}\right)>\varepsilon$ ) at current point. This lead to significant speed-up ( $\sim \times 50$ w.r.t the full Fast-Marching computation $)$ of the method, while the precision in the fibers direction is preserved.

\section{Experimental Results}

\subsection{Real HARDI Data}

We use a human brain dataset obtained on a Siemens 3T Trio scanner, with isotropic resolution of $1.7 \mathrm{~mm}^{3}, 60$ gradient directions, a $b=1000 \mathrm{~s} / \mathrm{mm}^{2}$, seven $b=0 \mathrm{~s} / \mathrm{mm}^{2}$ images, $\mathrm{TE}=100 \mathrm{~ms}$ and $\mathrm{TR}=12 \mathrm{~s}$, GRAPPA factor of 2 and a NEX of 3. The data is corrected to subject motion.

From these HARDI measurements, the fiber ODF was reconstructed. As mentioned in the introduction, several fiber ODF reconstruction algorithm exist 12234 . Here, we used the analytical spherical deconvolution transform of the q-ball ODF using spherical harmonics [4]. We used an order 4 estimation with symmetric deconvolution fiber kernel estimated from the real data, resulting in a profile with $\mathrm{FA}=0.7$ and $[355,355,1390] \times 10^{-6} \mathrm{~mm}^{2} / \mathrm{s}$.

The geodesic tracking is performed within a white matter mask was obtained from a minimum fractional anisotropy (FA) value of 0.1 and a maximum ADC value of 0.0015 . These values were optimized to produce agreement with the white matter mask from the T1 anatomy. The mask was morphologically checked for holes in regions of low anisotropy due to crossing fibers.

\subsection{Geodesic Connectivity Results}

For each bundle except the Superior Longitudinal Fasciculus (SLF), experiments were carried out with $\rho(f)=\ln (f) / \ln (2), \varepsilon=1$ and $\alpha=2$ after thresholding values of the fODF under 1 to avoid negative values. Our method however demonstrates robustness w.r.t the exact choice of these parameters. Since SLF has high curvature, we set angular speed $\alpha=8$ in order to favor tracking of actual SLF rather than projections on the occipital cortex. Runtime was about $90 \mathrm{~min}$ for each bundle. It can be further reduced by computing only some of the connectivity maps, or by computing them only on a subset of white matter. While results presented below show connectivity maps on the full maps, experiments show that the bundles can be retrieved by stopping the algorithm when $20 \%$ of the mask has been visited. The runtime is then reduced to about $14 \mathrm{~min}$.

Figure 1 shows connectivity measures and some geodesics obtained from different seeds manually placed into major fibers bundles, which agree with our knowledge of the white matter anatomy. Notice the correctness of the maps 

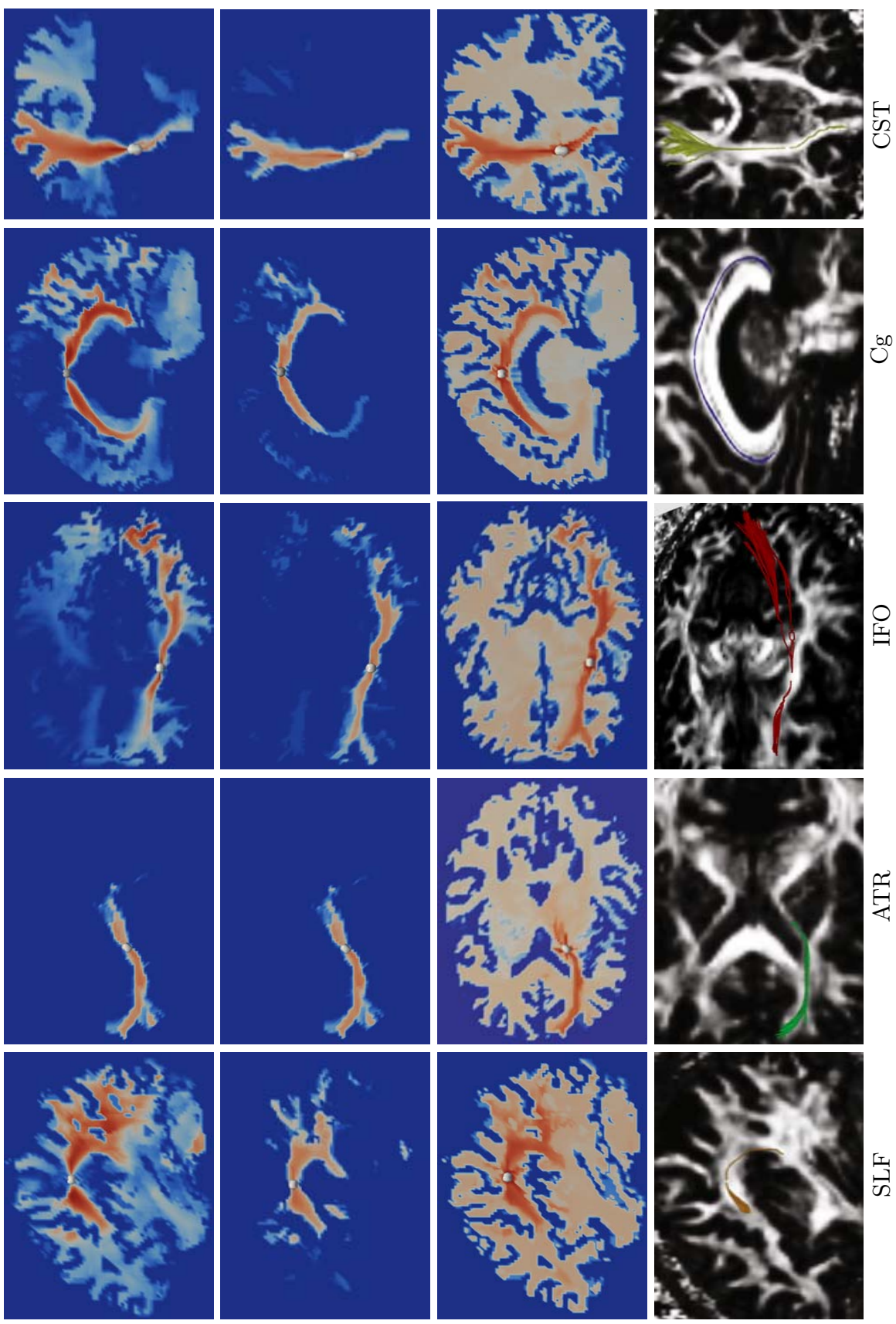

Fig. 1. Geodesic tracking results on five major fibers bundles. From left to right, $\mathcal{C}$, $\mathcal{C}_{\text {max }}, \mathcal{C}_{\text {sigma }}$ and some geodesics superimposed over the FA. 

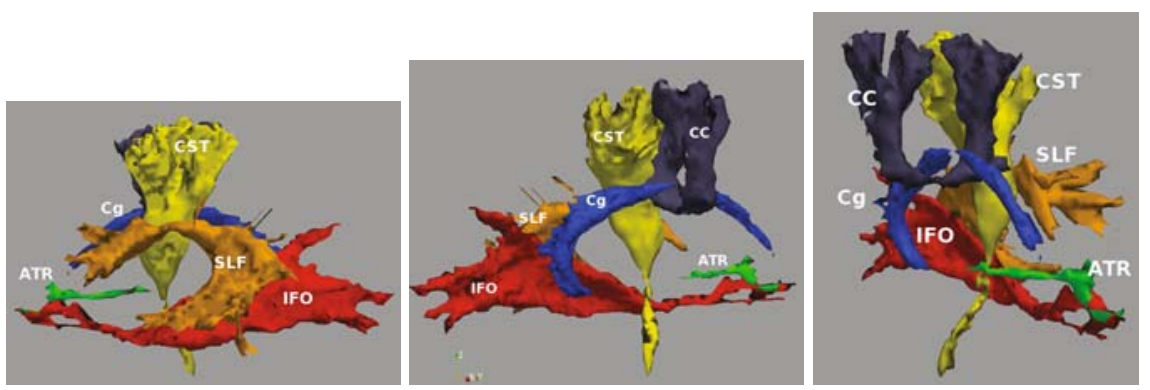

Fig. 2. Geodesic tracking results on major fibers bundles. We show isosurfaces of the connectivity measures of each bundle in a different color. In yellow, the CST; in blue, the $\mathrm{Cg}$; in red, the IFO; in orange, the SLF; in green, the ATR; in dark blue, a small part of the $\mathrm{CC}$ projections to the superior cortex.

on Corticospinal Tract (CST), which does not spread into the Corpus Callosum (CC). Also, the Cingulum (Cg), which is a thin structure close to $\mathrm{CC}$ is correctly handled by our method. This clearly shows the advantage of using a $5 \mathrm{D}$ space: since fibers in $\mathrm{Cg}$ and $\mathrm{CC}$ are perpendicular, these two bundles are very distant in our $5 \mathrm{D}$ space, while they are extremely close in $3 \mathrm{D}$. Other fibers bundles are also correctly retrieved, such as the Inferior Fronto-Occipital (IFO) fasciculus and the Anterior Thalamic Radiations (ATR). Furthermore, coherent results are obtained by the three proposed connectivity measures.

On figure 2 isosurfaces of the connectivity maps are shown for all the previous fibers bundles, as well as a small part of CC projections. Notice that CC is not segmented by our method. Rather, fibers are tracked from the given seed.

\section{Conclusion}

We presented a geodesic based tracking algorithm on HARDI data. Our method rapidly estimates connectivity maps inside a white matter mask from seed points, without the need for an explicit computation of fibers. Its versatility allows simultaneous computation of several different connectivity measures. Our experiments plead in favor of the use of a 5D space and show that our method is able to recover complex fiber bundles, which are often difficult to track.

\section{References}

1. Jansons, K.M., Alexander, D.C.: Persistent angular structure: new insights fom diffusion magnetic resonance imaging data. Inverse Problems 19, 1031-1046 (2003)

2. Tournier, J.D., Calamante, F., Connelly, A.: Robust determination of the fibre orientation distribution in diffusion MRI: Non-negativity constrained super-resolved spherical deconvolution. Neuroimage 35(4), 1459-1472 (2007)

3. Jian, B., Vemuri, B.C.: A unified computational framework for deconvolution to reconstruct multiple fibers from diffusion weighted MRI. IEEE Transactions on Medical Imaging 26(11), 1464-1471 (2007) 
4. Descoteaux, M., Deriche, R., Knösche, T.R., Anwander, A.: Deterministic and probabilistic tractography based on complex fibre orientation distributions. IEEE Transactions in Medical Imaging 28(2), 269-286 (2009)

5. Kreher, B.W., Schneider, J.F., Mader, J., Martin, E., Hennig, J., Il'yasov, K.A.: Multitensor approach for analysis and tracking of complex fiber configurations. Magnetic Resonance in Medicine 54, 1216-1225 (2005)

6. Bergmann, Ø., Kindlmann, G., Peled, S., Westin, C.F.: Two-tensor fiber tractography. In: ISBI, Arlington, Virginia, USA, pp. 796-799 (2007)

7. Wedeen, V., Wang, R., Schmahmann, J., Benner, T., Tseng, W., Dai, G., Pandya, D., Hagmann, P., D'Arceuil, H., de Crespigny, A.: Diffusion spectrum magnetic resonance imaging (dsi) tractography of crossing fibers. Neuroimage 41(4), 1267 1277 (2008)

8. Parker, G.J.M., Alexander, D.C.: Probabilistic anatomical connectivity derived from the microscopic persistent angular structure of cerebral tissue. Philosophical Transactions of the Royal Society, Series B 360, 893-902 (2005)

9. Perrin, M., Poupon, C., Cointepas, Y., Rieul, B., Golestani, N., Pallier, C., Riviere, D., Constantinesco, A., Bihan, D.L., Mangin, J.F.: Fiber tracking in q-ball fields using regularized particle trajectories. In: Christensen, G.E., Sonka, M. (eds.) IPMI 2005. LNCS, vol. 3565, pp. 52-63. Springer, Heidelberg (2005)

10. Seunarine, K.K., Cook, P.A., Embleton, K., Parker, G.J.M., Alexander, D.C.: A general framework for multiple-fibre pico tractography. In: Medical Image Understanding and Analysis (2006)

11. Behrens, T.E.J., Johansen-Berg, H., Jbabdi, S., Rushworth, M.F.S., Woolrich, M.W.: Probabilistic diffusion tractography with multiple fibre orientations. what can we gain? Neuroimage 34(1), 144-155 (2007)

12. Savadjiev, P., Campbell, J.S.W., Descoteaux, M., Deriche, R., Pike, G.B., Siddiqi, K.: Labeling of ambiguous sub-voxel fibre bundle configurations in high angular resolution diffusion MRI. Neuroimage 41(1), 58-68 (2008)

13. Zhang, F., Hancock, E.R., Goodlett, C., Gerig, G.: Probabilistic white matter fiber tracking using particle filtering and von mises-fisher sampling. Medical Image Analysis 13(1), 5-18 (2008)

14. Melonakos, J., Mohan, V., Niethammer, M., Smith, K., Kubicki, M., Tannenbaum, A.: Finsler tractography for white matter connectivity analysis of the cingulum bundle. In: Ayache, N., Ourselin, S., Maeder, A. (eds.) MICCAI 2007, Part I. LNCS, vol. 4791, pp. 36-43. Springer, Heidelberg (2007)

15. Lenglet, C., Prados, E., Pons, J., Deriche, R., Faugeras, O.: Brain connectivity mapping using riemannian geometry, control theory and pdes. SIAM Journal on Imaging Sciences 2(2), 285-322 (2009)

16. Jbabdi, S., Bellec, P., Toro, R., Daunizeau, J., Pelegrini-Issac, M., Benali, H.: Accurate anisotropic fast marching for diffusion-based geodesic tractography. International Journal of Biomedical Imaging, 1-12 (2008)

17. Jonasson, L., Bresson, X., Hagmann, P., Thiran, J., Wedeen, V.: Representing Diffusion MRI in 5D Simplifies Regularization and Segmentation of White Matter Tracts. IEEE Transactions on Medical Imaging 26, 1547-1554 (2007)

18. Sethian, J.A.: Level Set Methods and Fast Marching Methods. Cambridge University Press, Cambridge (1999)

19. Deschamps, T., Cohen, L.: Fast extraction of minimal paths in 3D images and applications to virtual endoscopy. Medical Image Analysis 5(4), 281-299 (2001) 